\title{
Abbreviazione bibliografiche
}

AA

AIIN

AJA

ALMA

AMAP

AnalBolland

Analysis Archeologica

AnnInst

AnnFaina

Antiqua et Mediaevalia

AntTard

AquilNost

$\mathrm{ArchCl}$

Arctos

Aristonothos

ASAA

Athenaeum

AttiMemTivoli

BABesch

BAntFr

BCom

$B d A$

Bullinst

BNum

Boreas

BSFN

BCTH

Cahiers Glotz

Chiron

$\mathrm{Civ} \mathrm{ClCr}$
Archäologischer Anzeiger

Annali dell' Istituto Italiano di Numismatica

American Journal of Archaeology

Archivum Latinitatis Medii Aevi

Atti e memorie della Accademia Petrarca di Lettere, Arti e Scienze

Analecta Bollandiana. Revue critique d'hagiographie

Analysis Archaeologica. An International Journal of Western Mediterranean Archaeology

Annali dell'Instituto di Corrispondenza Archeologica

Annali della Fondazione per il Museo Claudio Faina

Anuari de Filologia. Antiqua et Mediaevalia

Antiquité tardive. Revue internationale d'histoire et d'archéologie

Aquileia nostra

Archeologia classica.

Arctos. Acta philologica Fennica

Aristonothos. Scritti per il Mediterraneo antico

Annuario della Scuola Archeologica Italiana di Atene e delle Missioni Italiane in Oriente

Athenaeum. Studi di letteratura e storia dell'antichità

Atti e memorie della Società tiburtina di storia e d'arte

Bulletin Antieke Beschaving

Bulletin de la Société nationale des antiquaires de France

Bullettino della Commissione Archeologica Comunale di Roma

Bolletino d'Arte

Bullettino dell' Instituto di Corrispondenza archeologica Bollettino di numismatica

Boreas. Münstersche Beiträge zur Archäologie

Bulletin de la Société Française de Numismatique

Bulletin Archéologique du Comité des travaux historiques et scientifiques

Cahiers du Centre Gustave Glotz

Chiron. Mitteilungen der Kommission für Alte Geschichte und Epigraphik des Deutschen Archäologischen Instituts Civiltà Classica e Cristiana 
Abbreviazione bibliografiche

Daidalos

Epigraphica

EtrSt

Eutopia

Gerión

Hermes

Hesperia

Hortus ArtiumMedievalium

Instrumentum

$J D A I$

$J S$

JWCI

Latomus

LIMC

Mediterranea

MEFRA

MemAmAc

MonInst

Mythos

Nikephoros

NSc

Orizzonti

OudhMeded

Palladio

Pallas

ParPass

PBSR

Picus

PAPhS

Prospettiva

$\mathrm{RACr}$

RAN
Daidalos. Studi e ricerche del Dipartimento di science del mondo antico

Epigraphica. Periodico internazionale di epigrafia

Etruscan Studies. Journal of the Etruscan Foundation

Eutopia. Commentarii novi de antiquitatibus totius Europae

Gerión. Revista de Historia Antigua

Hermes. Zeitschrift für klassische Philologie

Hesperia. Journal of the American School of Classical Studies at Athens

Hortus Artium Medievalium. Journal of the International Research Center for Late Antiquity and Middle Ages

Instrumentum. Bulletin du Groupe de travail européen sur l'artisanat et les productions manufacturées dans l'Antiquité

Jahrbuch des Deutschen Archäologischen Instituts Journal des Savants

Journal of the Warburg and Courtauld Institut

Latomus. Revue d'études latines

Lexicon Iconographicum Mythologiae Classicae

Mediterranea. Quaderni annuali dell'Istituto di studi sul Mediterraneo antico

Mélanges d'Archéologie et d'Histoire de l'école Française de Rome, Antiquité

Memoirs of the American Academy in Rom

Monumenti inediti dell'Instituto di Corrispondenza Archeologica, Roma

Mythos. Rivista di storia delle religioni

Nikephoros. Zeitschrift für Sport und Kultur im Altertum

Notizie degli scavi di antichità

Orizzonti. Rassegna di archeologia

Oudheidkundige mededelingen uit het Rijksmuseum van

Oudheden te Leiden

Palladio. Rivista di storia dell'architettura

Pallas. Revue d'études antiques

La parola del passato

Papers of the British School at Rome

Picus. Studi e ricerche sulle Marche nell'antichità

Proceedings of the American Philosophical Society

Prospettiva. Rivista di storia dell'arte antica e moderna

Rivista di Archeologia Cristiana

Revue archéologique de Narbonnaise 
REL

RendLinc

RendPontAc

RH

$R M$

RSP

Saguntum

ScAnt

SEBarc

Spoletium

StEtr

StMisc

Temporis Signa

VeteraChr

ZKuGesch

ZPE
Revue des études latines

Atti dell'Accademia nazionale dei Lincei: Rendiconti

Rendiconti della Pontificia Accademia Romana di Archeologia

Revue historique

Mitteilungen des Deutschen Archäologischen Instituts, Römische Abteilung

Rivista di Studi Pompeiani

Saguntum. Papeles del Laboratorio de Arqueología de València

Scienze dell' Antichità: Storia, archeologia, antropologia Sylloge Epigraphica Barcinonensis

Spoletium. Rivista di arte, storia, cultura

Studi Etruschi

Studi miscellanei. Seminario di archeologia e storia dell'arte greca e romana della Università di Roma

Temporis Signa. Rivista di archeologia della tarda antichità e del medioevo

Vetera christianorum

Zeitschrift für Kunstgeschichte

Zeitschrift für Papyrologie und Epigraphik 
